\title{
Los criterios en la selección, secuenciación y organización de los contenidos de biología: una mirada de los docentes de secundaria en Barrancabermeja
}

\section{Criteria for selecting, sequencing and organization of the contents of biology: an look for teachers in secondary Barrancabermeja.}

Silvio Daza Rosales ${ }^{1}$, José R. Anrieta Vergara ${ }^{1}$, Oswaldo Ríos Canascal', Carlos Crespo Rojas ${ }^{1}$, Alfonso Torres Duarte ${ }^{1}$, Lizett Becenra Gonzales ${ }^{2}$ y Nery Galván Gonzales ${ }^{2}$.

1 . Instituto Universitario de la Paz (UNIPAZ). Miembros del grupo investigación para la renovación de la enseñanza de las ciencias (GRECI). Barrancabermeja. Colombia. ${ }^{2}$ Estudiantes $X$ semestre licenciatura Didáctica de la Ciencias Naturales de la UNIPAZ y del semillero de investigación (GRECI).biosidaza@hotmail.com llanadero@hotmail.com; osw62@hotmail.com; caliche crespo@hotmail.com; alfonsotorresduarte@hotmail.com .

\section{Resumen}

Este trabajo permite conocer las ideas que los docentes de biología tienen sobre la secuenciación y selección de los contenidos importantes o relevantes que deben enseñar y definir las relaciones que deben establecerse en el desarrollo de los mismos en un momento dado y a lo largo del tiempo académico; de igual manera se trató de determinar los criterios que los docentes tienen en cuenta para la selección y secuenciación de los mismos.

La investigación se desarrolló con 22 docentes de Biología de los colegios de secundaria de la ciudad de Barrancabermeja, Santander, fue un estudio de tipo descriptivo. Para la toma de información se utilizó una encuesta estructurada, se revisaron los preparadores de clases y seis planes de áreas de los colegios de la ciudad y la hipótesis que se planteó fue que los docentes de Biología seleccionan y secuencian los contenidos de enseñanza, de la misma manera como se los presentaron en su formación profesional.

En la investigación se observó que los docentes seleccionan y secuencian los objetivos teniendo en cuenta el contenido disciplinar, los libros de textos y la edad de los estudiantes; en ellos predomina la concepción que estudiantes de igual edad presentan las mismas capacidades e intereses y no tienen en cuenta la influencia del contexto social donde se desenvuelven, de igual manera no le dan importancia al papel de las ciencias en la vida de las personas y el conocimiento enseñado es totalmente descontextualizado y solo basado en lo que le proponen los libros de texto.

Palabras claves

Contenidos, Seavenciación, disciplina, Conocimiento Escolar, Curníaulo. Biología.

\section{Abstract}

This work allows to know the ideas of biology teachers have on the sequencing and selection of important or relevant content they must teach and define the relationship to be established in developing them at a given time and over time academic; was treated similarty to determine the criteria that teachers have in mind for the selection and sequencing of the same.

The research was developed with 22 biology teachers from secondary schools in the city of Barrancabermeja, Santander, was a descriptive study. In making information uses a structured 
survey, we reviewed the preparation of classes and six area plans of the schools in the city and the hypothesis raised was that biology teachers selected and sequenced by the contents of teaching, same way as they are presented in their training.

In the research note that teachers are selected and sequenced objectives taking into account the content discipline, textbooks and student age and in these predominant conception of the same age students have the same abilities and interests and have into account the influence of social context in which they develop, just as they do not give importance to the role of science in the lives of people and knowledge taught is totally out of context and only based on what the textbooks suggest.

\section{Keywords}

Content, Sequencing, Discipline, Knowledge School Curriculum, Biology

\section{Introduccion}

Iniciado el siglo XXI, se puede preguntar ¿qué ha cambiado en la enseñanza de las ciencias? ¿Prevalecerán los mismos conceptos de escuela, aprendizaje y currículo; o estarán orientados a satisfacer los requerimientos de la época actual?. No es extraño ver que las ciencias aun sigan siendo tratadas como un arerpo inalterable de conocimientos preexistentes. $Y$ esto, se ve reflejado en los curnículos de ciencias, que siguen dominados por la exigencia de comprender y asimilar un extenso cuerpo de conocimientos factuales que se consideran un requisito esencial para la formación en ciencias. Aún, en esta época se sigue con ideas del positivismo empírico, donde se supone que el mundo tiene una realidad y el aprendizaje consiste en tratar de aprender de ella y donde se considera el proceso de investigación como una receta de carácter cient́fico, que responde al mismos formato en cualquier tipo de ciencia.

Bajo este paradigma, el papel del profesor y de quienes producen planes de estudio, textos y materiales didácticos ha consistido en diseñar estrategias curriculares y didácticas, que faciliten a los estudiantes la asimilación del conocimiento transmitido. Esta es la educación que a través del tiempo se ha caracterizado en las instituciones educativas, que imparten los docentes actualmente y que van en contrariedad con la epistemología constructivista que se quiere acoger en la actualidad.

La epistemología de las ciencias, en su versión modema, propone el estudio de la naturaleza del conocimiento y de las circunstancias de su producción. De ahí que resulte importante la toma de conciencia, por parte del educador, de sus creencias en relación al mismo y la forma como éste se genera; las relaciones entre el conocimiento y la realidad y entre las distintas manifestaciones del saber científico, de modo que el educador pueda emplear de manera explíata estas ideas en el diseño de su acción pedagógica.

Por consiguiente, uno de los desafíos de los docentes no es acercar a los alumnos al campo de conocimiento científico sino al escolar; que "es el conjunto de conocimientos a enseñar y de aprendizajes a construir a través de una educación cient́fica formal, sistemática y organizada, en donde los estudiantes alcancen niveles deseables de alfabetización científica, lo cual se puede lograr a partir de las tres dimensiones de los contenidos: el teórico, el procesual y el actitudinal ${ }^{1}$, que se estructura en el modelo actual de ciencias(Liguori, 2005).

Este desafío contrasta con las nuevas demandas, que se hacen a los docentes desde una concepción constructivista del aprendizaje. Debido a que el educador debe desempeñar un nuevo rol y estar en capacidad de desarrollar un currículo y diseñar unidades didácticas a través del trabajo cooperativo, teniendo en cuenta aspectos; cómo estructurar la dase; de donde obtener los 
citerios para poder seleccionar los contenidos; cómo secuenciarlos; a que problema apuntar; los objetivos que se quieren lograr o qué modelo tener en cuenta para conocer los errores y dificultades de los estudiantes. De igual manera, el docente tiene la responsabilidad profesional de secuenciar y seleccionar los contenidos que caracteriza la disciplina; lo importante o relevante que debe enseñar. Puesto que, "el contenido es el conjunto de formas culturales y de saberes seleccionados para formar parte de las distintas áreas curriculares en función de los objetivos generales de área, los contenidos pueden ser hechos discretos, conceptos, principios, procedimientos, valores, normas y actitudes, ayya seavenciación requiere analizar los aspectos que se consideran más relevantes, y definir las relaciones que deben establecerse en su desarrollo en un momento dado y a lo largo del tiempo (Del Carmen Martin, 2005).

\section{Planteamiento del problema}

La enseñanza de las ciencias naturales ha despertado $-y$ continúa despertando- opiniones críticas en relación a sus contenidos, como de la metodología utilizada por los docentes para transmitir dichos contenidos. Según (Pozo, 2004), esto hace parte del quehacer cotidiano y se presenta cuando los docentes tienen que llevar a término los objetivos que plantea la enseñanza de las ciencias en cada nivel y en cada país; seleccionan diversos contenidos, programan diversas actividades a realizar con los estudiantes, preparan materiales y recursos que utilizan en el aula y toman decisiones sobre el qué y como enseñar y cómo hacerlo.

Uno de los principales obstáculos, al momento del proceso de enseñanza, es el poco conocimiento y actualización de los maestros con respecto a los contenidos escolares; (Reyes, 1998), en una investigación realizado con 322 docentes de ciencias naturales de Colombia, mostro que el $\mathbf{8 0} \%$ de las personas entrevistadas seleccionaban contenidos teniendo en cuenta los programas académicos, un $18 \%$ cambiaba el orden de los temas y un $2 \%$ introducían temas científicos de la actualidad.

Según lo anterior, los conocimientos que pretenden enseñar los docentes se convierten en contenidos independientes y con el mismo nivel de complejidad; poco importa el ordenamiento y la distribución, porque siguen la lógica de la disciplina , el que proponen los textos o el orden estricto que han seguido los cient́ficos en la construcción de conocimientos a lo largo de la historia, es mas, como señala (Izquierdo, 2005), lo que enseña el docente en las aulas dífiere significativamente del curnículo oficial.

Un estudio realizado por (Sánchez y Valcárcel, 2000) sobre los criterios que tenían en cuenta los docentes para seleccionar contenidos mostro que el $70 \%$ de estos tenían en cuenta el contenido disciplinar, el $\mathbf{8 1 \%}$ por el tiempo que tenían para desarrollar el programa, el 52\% utilizaban las planificaciones anteriores, un $\mathbf{5 2 \%}$ los programas oficiales, un $\mathbf{4 8 \%}$ los libros de textos y un $\mathbf{4 8 \%}$ indico que el contenido que utilizaban para la enseñanza era exdusivamente teórico y tomaban su experiencia docente como fuente del conocimiento sobre el alumno.

Estas referencias y las observaciones de aula por parte de estudiantes de licenciaturas en didáctica de las ciencias naturales mostraron que el profesorado de las asignaturas de ciencias, de las diferentes instituciones de Barrancabermeja, utilizan poco los libros de texto, hacían mucho énfasis en la enseñanza de los conceptos, sin hacer mucho énfasis en las actitudes y procedimientos, por lo que seria muy prudente analizar si la secuencia y la selección de los contenidos de Biología se realizan teniendo en cuenta los aprendizajes, que son muy importantes y las relaciones que se establecen entre los nuevos conocimientos y los que el alumno ya tiene o, por lo contrario, el docente no distingue la importancia de las características especificas de cada alumno en el proceso de enseñanza. 
Por lo tanto, se requería indagar qué es lo que lleva a los docentes a seleccionar los contenidos para la enseñanza de la biología; ya que la transformación educativa demanda la adecuación de los contenidos, del cómo enseñar, qué y cómo evaluar el trabajo escolar si se quiere plantear un enfoque integrador de carácter interdisciplinario que propicie la integración del conocimiento cientúfico y el cotidiano. por lo aual formulamos la siguiente pregunta ¿Cuáles son los criterios que utilizan los docentes de secundaria de los colegios de Barrancabermeja, para la selección y secuenciación de contenidos de Biología?

\section{Marco teórico}

Apoyarse en la historia de las ciencias para transmitir la idea de que la ciencia es una construcción de conocimientos para resolver problemas, es una metodología útil y fructífera si se maneja adecuadamente. No es necesario insistir excesivamente en este aspecto, que ya ha sido tratado abundantemente y que ha sido resumido por (Estany, 2005).

La imagen de la ciencia que se transmite en la universidad a los futuros profesores - como unos contenidos ya hechos, como una serie de leyes que se deducen lógicamente a partir de unos principios- es, si no la razón fundamental, sí al menos una razón preponderante de distorsión en la comprensión de la ciencia (Daza y Arrieta 2006). No obstante, caben otras altemativas para no caer en ese enfoque, que parece deformado y deformante.

Según Daza y Arrieta (2002), existe un marcado contraste entre el aprendizaje de los estudiantes en Biología y las creencias de los educadores, ya que existe una fuerte coherencia entre la forma en que el maestro enseña y sus concepciones acerca de la disciplina, la enseñanza y el aprendizaje. Esta situación unida a la dificultad de cambio presentada por algunos docentes es persistente en todos los niveles de educación, desde el preescolar y la educación superior. Entender el pensamiento del maestro es uno de los factores críticos para proveer y promover mejores ambientes de aprendizaje en la enseñanza de la ciencias (Porlán, y Martín 1994).

Tradicionalmente las ciencias -ya sea con sus denominaciones disciplinares o enlatadas pero conservando los ingredientes bajo epígrafe de ciencias de la naturaleza- han ocupado un lugar importante en la educación obligatoria. Su inclusión en el currículo, desde los primeros niveles del sistema educativo, puede justificarse por diversos motivos:

$>$ Las necesidades de una sociedad en la que cada vez existe mayor desarrollo científico y tecnológico.

$>$ La auriosidad del ser humano por conocer las características, la posibilidad y las limitaciones de su propio cuerpo.

$>$ La importancia, en una sociedad democrática, de que los ciudadanos tengan conocimientos suficientes para tomar decisiones reflexivas y fundamentales sobre temas cientúfico-técnicos de incuestionable transcendencia social.

$>\quad$ La creencia que es imprescindible una participación activa y consciente en la conservación del medio y del desarrollo sostenible.

$>$ El interés por crear hábitos saludables, personales y colectivos, que mejoren nuestra calidad de vida.

$>$ La conveniencia de transferir muchos de sus valores formativos a otros contextos y situaciones cotidianas.

Se podrían añadir otras contribuciones de esta área de conocimientos a la formación básica de los estudiantes y posiblemente serian compartidas por gran parte del profesorado de ciencias. No obstante, también hay auestiones en las que las respuestas no son tan homogéneas: ¿Creemos que todos los contenidos de nuestras asignaturas están orientados al logro de esas intenciones 
educativas?; ¿Qué preocupa más, la adquisición de estos <<valores formativos>> o la explicación de la mayor parte del programa?; ¿Es la ciencia que impartimos la única o la más importante fuente de formación <<vital>> de los adolecentes que tenemos en el aula?; ¿Deben compartir que nuestra asignatura es la más importante?; ¿Por qué el alumnado, en general, le gusta cada vez menos las ciencias?

Conviene recordar que no es posible (ni parece necesario) que un estudiante sea capaz de acumular o reconstruir todo el conocimiento que hay actualmente sobre una materia, ni siquiera arando acaba su formación universitaria. Por lo tanto, siempre estaremos obligados a seleccionar un subconjunto de los que sabemos; es decir, algo, que nos resulta interesante 0 apasionante y que nos gustaría comunicar a nuestros alumnos, tendrá que esperar a otra ocasión.

Pero, con respecto al hilo de esta reflexión sobre la obligada selección del contenido de enseñanza, queremos plantear lo siguiente:

$>\mathrm{Si}$ los programas de las asignaturas vienen impuestos, directa 0 indirectamente, por alguien 0 por algo, ¿Estamos dispuesto a enseñar contenidos que, por nuestra experiencia profesional, consideremos inadecuado?; ¿Asumimos la validez del conocimiento de los libros de textos 0 otros materiales de aprendizaje sin cuestionar su idoneidad científica?

> Si la elección depende de nosotros, ¿Qué conocimientos cientúficos debeńamos enseñar?; ¿Nos centrańamos en la ciencia actual (más compleja pero más próxima a los problemas de la calle) o en <<la de siempre>> (menos comprometida pero en la que nos sentimos más seguros)?.

Los profesores tratan a veces de impartir programas sobrecargados de contenidos y se quejan de no tener tiempo suficiente para explicarlos. Si no les da tiempo de enseñarlos, ¿Cómo le va a dar tiempo al estudiante de aprenderlo?... paradójicamente, mientras más se avanza en las respuestas, mas preguntas surgen. Quizás lo único que queda daro sea que la enseñanza de las ciencias es compleja. Por lo que conviene entonces, empezar por el principio, los enfoques y métodos en los que se fundamentan e implícitamente con llevan a la secuenciación del contenido.

En general, la interacción entre profesores y alumnos se ve necesaria pero ¿qué pasa con los contenidos? ¿Verdad que pensamos en ellos como algo que 'está ahí' (en el libro de texto, quizás) y que, por más que hagamos, no cambia, sino que los profesores son mediadores entre ellos y los alumnos? Sin embargo, si aceptamos un enfoque sistémico para la dase, hemos de considerar que los contenidos intervienen en el juego y se configuran de la manera adecuada a la finalidad educativa que se persigue.

Si la finalidad es tan nueva y revolucionaria como la que ahora se nos está planteando (enseñar ciencias a/ para la ciudadanía) y tan diferente a la que dio origen a las disciplinas universitarias en las que nos hemos formado los profesores de ciencias, los contenidos de la ciencia escolar deberían ser igualmente nuevos y revolucionarios y la función del profesor, mucho más compleja que la de aproximar al estudiante unas disciplinas universitarias preestablecidas.

¿Es legítimo pensar en unas nuevas ciencias que ya no sean estrictamente las disciplinas actuales? Creo que lo imponen los nuevos tiempos y las nuevas finalidades educativas, democráticas, que requieren una mirada amplia sobre todas las ciencias para formar mentes abiertas y a la vez, científicas, desde la infancia con sus propios centros de interés que ya no corresponden a ninguna disciplina en exdusiva. ¿Cuáles son los límites para la interdisciplinaridad, transdisciplinaridad, multidisciplinaridad o como queramos llamar a esta visión de conjunto pero con sus temas propios? ¿Hay algo a lo que no podemos renunciar, puesto que, de hacerlo, ya no enseñańamos ciencias y, si lo hay, qué es? Para intentar responder a estas preguntas desde la visión sistémica de la clase que se propone, significa que se debe guiar también por las 
aportaciones de las ciencias cognitivas, en la versión filosófica- histónica en la que he trabajado muchos años y que proporciona un marco teórico que parece útil.

Las ciencias son el resultado de una 'actividad humana' con una finalidad científica (que es una actividad humana, para el científico), en la cual confluyen el pensamiento, el lenguaje y la experimentación y gracias a la cual emergen nuevos conocimientos. Parten de una vivencia, a partir de la cual surgen preguntas y se promueven respuestas. Sin embargo, estas preguntas y estas respuestas, si han de ser auténticas, no pueden ser las que se susciten en una clase de ciencias para el ciudadano. Quizás por ello la ciencia 'disciplinar' no genera 'experiencia vital' y por lo tanto no funciona desde un punto de vista cognitivo. Las reflexiones de filósofos de la ciencia cognitivos, como (Giere, 1992), nos aportan ideas para avanzar hacia una epistemología propia de la ciencia escolar, con la cual ésta adquiere la independencia que necesita, sin dejar de cumplir los requisitos fundamentales de la educación científica.

Es decir, va ayudar a responder a las preguntas que planteábamos al inicio de este apartado, estableciendo como requisito fundamental la adquisición del pensamiento teórico (aprender a pensar mediante modelos del mundo) para explicar las intervenciones experimentales en el mundo. Ahora bien, ambos (modelos, lenguaje y experimentos) han de ser los propios de la ciencia escolar, no los de la ciencia universitaria sin más.

\section{Metodologìa}

Esta investigacion se realizo con 22 docentes de secundaria del sector oficial en la asignatura de biología del municipio de Banancabermeja, fue un estudio de tipo descriptivo que busco describir de forma objetiva los hechos que tiene lugar en una población determinada.

La hipótesis de investigación fue que los docentes de secundaria de Barrancabermeja del área de biología seleccionan y secuencian los contenidos teniendo en cuenta las leyes educativas. Los libros de texto y utilizando como eje central los conceptos que caracterizan la disciplina que ejercen, relegando a un segundo plano la adquisición de procedimientos y vivencia de actitudes, por lo que se planteo como objetivo conocer los criterios que tiene en auenta para seleccionar y secuenciar los objetivos.

Se aplicaron encuestas, se realizaron entrevistas y se revisaron documentos docentes tales como diarios de campo, parceladores y planeadores y los datos se organizaron numéricamente por medio de tablas de frecuencias y por medio de graficas.

\section{Resultados y discusión}

Contenido disciplinar. Las respuestas de los docentes muestran que, aunque estos tengan en arenta sus concepciones para desarrollar sus temas, no reflejan un modelo didáctico determinado y plantean su estructura teniendo en cuenta el contenido disciplinar.

La programación anual se realiza utilizando como elemento dave el contenido disciplinar y referenciándose en, las planificaciones de años anteriores, la experiencia docente, y estos mismos aspectos se tienen en cuenta para modificar la estructura de los contenidos con el fin de simplificar la materia, incidir más en algunos contenidos o fijar contenidos mínimos. Por lo que se puede decir que existe una situación no deseable, por lo que se hace necesaria que se considere el arestionamiento de sus concepciones y practicas habituales, ya que esto posibilitańa que sus hábitos de planificación sean fundamentados y se estructuren adecuadamente los contenidos. 
El eje estructurador para organizar el contenido, que los docentes afirman tener tampoco esta definido. Por lo que puede deducirse que no se encuentran Informados sobre la manera de utilizar los diferentes tipos, ya que "existe una amplia producción teórica e investigaciones que han demostrado que no es posible el aprendizaje de procedimientos científicos sin el aprendizaje de conceptos". Si bien esta forma de definirlos ha sido objeto de interesantes discusiones en ciertos ámbitos académicos, esta ausente en la mayoría de los diseños curriculares, y más aun en el espíritu de los formadores de docentes y en la mayoría de maestros y profesores. De manera, se ha elevado la importancia de la categoría de contenido de los procedimientos, valores, actitudes, etc., lo aul implica preocuparse por como y cuando se los va enseñar, y por como se los va a evaluar. Implica también la necesidad de reflexionar acerca de cómo se aprenden los procedimientos o los valores, y cuales podrían ser las estrategias de enseñanza mas adecuada en cada caso.

Libros de texto. Es otro referente consultados por los profesores, como lo pueden ser la internet, Encarta y artículos de revista. Así mismo, afirman utilizar editoriales como son: Santillana, Voluntad y Norma. Por tanto, lo que muchos docentes no se percatan es que textos escolares publicados en años recientes, "repiten saberes o propuestas de actividades muy parecidos a las que el maestro aprendió en sus años de formación". Esos contenidos resultan "familiares"; por lo que no lo desestructuran y sienten que el texto escolar, a diferencia del diseño curricular, lo ayuda, lo organiza.

Los textos escolares de ciencias, poseen unos contenidos, los auales deben ser presentados acorde a los conceptos propios de la ciencia, induyendo aspectos como la terminología cientíica, actualización de sus contenidos, profundidad en los contenidos y pertinencia a la edad de los estudiantes a la que va dirigido el texto y con una correcta interpretación de la epistemología de la ciencia". Sin embargo, al seleccionar el contenido a través de este referente los docentes lo condicionan como guía para un curso en espeáfico y como apoyo en niveles superiores, sobre todo en bachillerato. De igual forma, realizan modificaciones de las que se refieren a la reducción de contenidos a su simplificación o al cambio de una definición por otra que les parezca más adeauada. Además, en ningún caso mencionan criterios cient́ficos o didácticos para explicar su selección del contenido o las modificaciones que introducen en el libro de texto, más aun la naturaleza del contenido de enseñanza que los docentes plantean son contenidos teóricos coherentes con el carácter conceptual de los libros.

Edad de los estudiantes. Investigaciones muestran que los alumnos no llegan en blanco a cada nueva situación de aprendizaje escolar, si no portando esquemas de conocimientos previos. Estos esquemas constituyen representaciones de la realidad, y en ellos se articulan tanto conceptos construidos en el ámbito escolar como otros construidos espontáneamente en la practica extraescolar cotidiana".

los docentes al momento de seleccionar y secuenciar los contenidos de enseñanza, pocas veces tienen en cuenta la edad cronológica de los estudiantes y por lo tanto el nivel cognitivo de estos en contravía a Coll y Martin (1990) que señalan que "los niveles de desarrollo identificados por la sicología genética, que permiten definir lo que un individuo puede comprender, hacer 0 aprender en un momento determinado, son útiles para seleccionar y establecer las secuencias de los contenidos que pretender enseñarse". Por tanto, las secuencias induidas en una determinada etapa educativa debeńan enlazar con los conocimientos previos de los alumnos, para garantizar la progresión en los procesos de aprendizaje que realizan los alumnos. Por otro lado, y ya dentro de cada secuencia, es conveniente que se induyan, a lo largo de la misma, resúmenes y recapitulaciones que permitan recordar en un momento dado el camino recorrido en la secuencia de aprendizaje, para que los alumnos puedan seguir su hilo conductor, y a la vez recordar aquellos contenidos necesarios sobre los que construir los siguientes aprendizajes. 


\section{Conclusiones}

$>$ Los criterios utilizados para planificar los contenidos de Biología solo haćan referencia al contenido disciplinar, el libro de texto y la edad de los estudiantes; el docente cree que todos los estudiantes de la misma edad, tienen las mismas capacidades y los mismos intereses y no tienen en cuenta el desarrollo social de estos y por tanto, el entomo en el que se desenvuelven. Así mismo, le restan importancia al desarrollo de las ciencias en la vida de las personas, y le brindan gran importancia a los temas planteados en los libros de texto donde se les dice que contenido se debe enseñar y cómo deben hacerlo llegar al estudiante.

> La revalorización de los contenidos conceptuales, no es proveniente solamente de una enseñanza basada en procesos . esta debe ir encaminada hacia una nueva conceptualización acerca del propósito de la enseñanza escolar, dode se resalte la intencionalidad de promover en los alumnos la construcción de esquemas de conocimiento. En esta fase, se puede decir que la transmisión de contenidos conceptuales desempeña un rol importante en el proceso de construcción de conocimientos; donde este es el que debe ser transmitido por la escuela de modo tal que se garantice su aprobación activa (significativa) por parte de los alumnos.

> Un curriculo educativo con unos objetivos amplios, orientados al desarrollo global y amplio, no puede nutrirse de una unica fuente sin correr el riesgo de marginar aspectos importantes desde el punto de vista educativo. El referente disciplinar, que con frecuencia ha sido el unico tenido en cuenta apartir de la educacion secundaria, debe ser contemplado junto a otros de diferente naturaleza, ya que muchos contenidos educativos considerados relevantes desde una prespewctiva social, no estan oraganizados en disciplinas.

> Aunque el analisis de los contenidos se ha procupado por los aspectos conceptuales del conocimiento,varias de las aportaciones analizadas plantea la necesidad de contemplar simultaneamente y de manera iterrelacionada sus aspectos procedimentales y actitudinales.

> Se debe crear la necesidad de analizar los distintos tipos de contenidos que deben ser objeto de enseñanza: Conceptuales, procedimentales y actitudinales y sus interrelaciones, para que esto tenga un verdadero sentido en el aprendizaje de los estudiantes.

\section{Bibliografía}

Coll, C., y Martin, E. (1990). Aprendizaje y Desarrollo: La concepción genético-cognitiva del aprendizaje, en Coll, Palacios, Merchis (comps) Desarrollo Psicológico y Educación II: Psicología y educación. Madrid: Alianza.

Daza Rosales, S. y Arrieta Vergara, R. (2006). Los conceptos sobre ciencia y trabajo cientúfico y sus implicaciones en la elaboración de los programas de ciencias naturales. Tecné, Episteme $y$ Didaxis, No. 20, $80-95$.

Daza Rosales, S. y Arrieta Vergara, R. (2002). Los gráficos de los libros universitarios de Biología y su influencia en la producción de una concepción altemativa sobre la célula. Educación en ciencias e ingenierías. Universidad de la Paz.Vol.1.№ 2, 12-16.

Del Carmen Martín, L. M. (1995). Criterios para el análisis, selección, secuenciación y organización de los contenidos educativos en el curnículo: aplicación a la secuenciación de contenidos de Biología en la educación primaria. España, Universidad de Griona. Departamento de Psicología, Editora. 
Estany, A. (2005). El papel de la historia de la ciencia en los estudios interdisciplinares de la ciencia. En S.F. Martínez y G. Guillaumin (Comp.), Historia, filosofía y enseñanza de la ciencia (pp. 291-303). México: UNAM.

Izquierdo, M. (2005). Hacia una teońa de los contenidos escolares. Barcelona, España: Universitat Autònoma de Barcelona, Editora

Giere, R (1992). Cognitive Models of Science. Minneapolis: University of Minnesota Press.

Liguori, L. y Noste I. M. (2005). Didáctica de las Ciencias Naturales. Argentina: Homo Sapiens Editora.

POZO, J.I. Y GOMEZ CRESPO, M. A. (2004). Aprender y enseñar ciencia, Del Conocimiento Cotidiano Al Conocimiento Científico. Madrid, España: Morata Editora.

Porlán, R. y Martín, J. (1994). El saber práctico de los profesores especialistas. Aportaciones desde las didácticas específicas. Investigación en la Esavela, 24, pp. 49-58.

Reyes Herrera, L. (1998 la epistemología del maestro: Un poderoso referente en la enseñanza de la Biología. En: Teane, Episteme y Didaxis. № 4. p 27-36.

Sánchez Blanco, G. y Valcárcel Pérez, M. V. (2000). ¿Qué Tienen en Cuenta los Profesores Cuando Seleccionan el Contenido de Enseñanza? Cambios y Dificultades tras un Programa de Formación. En: Enseñanza de las Ciencias: 18 (3), 423-437. Barcelona 